\title{
Bilateral Neurovascular Bundles Sparing Prostatectomy Preserves Sexual Function in Patients with Localized Prostate Cancer
}

\author{
MAKOTO NAKIRI, MASANORI NOGUCHI, TATSUYA ISHITAKE** \\ AND KEI MATSUOKA \\ Departments of Urology and Environmental Medicine*, Kurume University School of Medicine, \\ Kurume 830-0011, Japan
}

Received 8 January 2009, accepted 16 February 2009

\begin{abstract}
Summary: The influence of the presence or absence of the neurovascular bundles on patient QOL were examined using the UCLA Prostate Cancer Index (UCLA-PCI) in patients who underwent radical retropubic prostatectomy. The study was performed in 105 patients who were histopathologically diagnosed with prostate cancer and underwent radical retropubic prostatectomy (During prostatectomy, the bilateral neurovascular bundles were preserved in 45 patients $(42.8 \%)$, unilateral neurovascular bundle preservation was achieved in 24 (22.9\%), and no neurovascular bundles were preserved in $36(34.2 \%))$. The QOL was evaluated before and after surgery using the Japanese edition of the UCLA-PCI, which examines 6 items. Our findings suggested that 'urinary function, 'urinary bother', 'bowel function', and 'bowel bother' deteriorated early after surgery, and recovered to the preoperative levels in the late phase after surgery, but no significant difference was noted in the time-course among the three groups. In contrast, 'sexual function' was significantly improved in the late postoperative phase only in the bilateral nerve-spared group, but not in the unilateral nerve-spared and non-nerve-spared groups. Patients complaining of 'Sexual bother' were more prevalent in the unilateral nerve-spared group in the late postoperative phase, but the difference was not significant. On multiple regression analysis of factors associated with sexual function in the late postoperative phase, only bilateral nerve preservation of was significantly associated with sexual function in the late postoperative phase $(\mathrm{p}<0.0001)$. In order to maintain sexual function following radical retropubic prostatectomy, the bilateral neurovascular bundles should be preserved, as far as practicable.
\end{abstract}

Key words nerve preservation, prostate cancer, QOL, radical retropubic prostatectomy, sexual function, UCLAProstate Cancer Index

\section{INTRODUCTION}

Prostate cancer is a rare cancer with regard to the concept of course observation without treatment. Considering its characteristic slow growth, the induction of severe, prolonged complications following surgery raises questions doubt the suitability of surgical treatment as compared to radiotherapy, and physicians have to obtain detailed informed consent before surgery, covering the possible occurrence and duration of complications, surgical procedure, and adverse effects.
Detection of localized prostate cancer has markedly increased in recent years with the spread of PSA screening, and radical retropubic prostatectomy is the global standard procedure, taking into consideration patient age and cancer stage. However, this procedure causes complications, such as dysuria (urinary incontinence) and sexual dysfunction (impotence), markedly impairing the patient's QOL in many cases. Of these complications, approaches to postoperative recovery from dysuria (urinary incontinence) have become consistent internationally. Hamada et al. reported that urination is in the recovery period 3

Corresponding Author: Makoto Nakiri, Department of Urology, Kurume University School of Medicine, 67 Asahi-machi, Kurume 830-0011, Japan. Tel: 0942-31-7552 Fax: 0942-31-4370 E-mail: mnakiri@med.kurume-u.ac.jp 
months after surgery, and returned to the preoperative level 6 months after surgery [1], indicating that recovery in the early postoperative phase can be expected. Our hospital has succeeded in early catheter removal, improvement of urinary incontinence, and discharge using Noguchi's anastomosed site-lifting method and by developing a clinical path [2]. In contrast, sexual dysfunction (impotence) occurs in more than half of patients after surgery, and their QOL is impaired for a long time after surgery. The outcome markedly varies among facilities, and at present no standard, consistent approach to preservation of sexual function has been developed, despite the many studies on the influence of nerve-sparing and age on postoperative sexual function that have been published after Walsh et al. initially reported nerve-sparing radical prostatectomy [3].

The UCLA Prostate Cancer Index (UCLA-PCI) is a localized prostate cancer-targeted QOL questionnaire widely used internationally, consisting of 20 items covering 6 aspects of complications following radical prostatectomy ('urinary function', 'urinary bother', 'bowel function', 'bowel bother', 'sexual function', and 'sexual bother'), and the reliability and validity of the Japanese edition has been confirmed [4]. In addition to the disease-targeted QOL questionnaire, FACT-P, EORTC, and SF-36 are generally used as health-related QOL questionnaires in other studies on QOL, but the UCLA-PCI covers typical complications following radical prostatectomy, and consists of only 20 items so is considered less stressful than other indices.

In this study, we evaluated the QOL of patients following radical retropubic prostatectomy for localized prostate cancer using the Japanese edition (version 1.2) of the UCLA-PCI, and investigated the influence of surgery on the patients' QOL, factors involved, changes in the QOL during the postoperative period, and the influence of preservation of the neurovascular bundles, hoping that the findings would prove useful information to patients considering radical prostatectomy.

\section{SUBJECTS AND METHODS}

The study was performed in 105 patients who were histopathologically diagnosed with prostate cancer and who underwent radical retropubic prostatectomy at Kurume University Hospital and 8 related facilities between January 2002 and February 2006. The neurovascular bundles were preserved in 69 patients (65.7\%) (bilateral: 45 (42.9\%), unilateral: 24
$(22.9 \%)$ ), and no nerves were preserved in 36 (34.3\%). Regarding nerve preservation, patient's requests were given a high priority, but in most cases the side positive on biopsy was resected. The bilateral nerves were resected in all cases that received concomitant MAB treatment. The localization of cancer in the prostate was confirmed by MRI. As a rule, the bilateral nerves were preserved in all cases after August 2005. The bilateral nerves were resected in all cases with concomitant MAB treatment, as a rule. The same operator performed all surgeries, employing the anastomosed site-lifting method via the retropubic approach developed by Noguchi [2]. The clinical stage was diagnosed following the 5 th edition of the TNM classification revised by the UICC in 1997. No postoperative adjuvant therapy, such as treatment with sildenafil citrate, was performed in any patient.

For evaluation of QOL before and after surgery, the Japanese edition (version 1.2) of the UCLA-PCI was used. The patients filled in the questionnaire privately, in the absence of physicians. The survey was performed before surgery and 1, 3, 6, and 12 months after surgery.

One and 3 months after surgery were designated as early postoperative phase, and 6 and 12 months after surgery was the late postoperative phase. In patients who could be surveyed in both phases, answers at 1 month were adopted as those in the early postoperative phase, and answers at 12 months as those in the late postoperative phase in the investigation of time-course changes. Complications were classified into 6 aspects following the international criteria: 'urinary function', 'urinary bother', 'bowel function', 'bowel bother', 'sexual function', and 'sexual bother', and were assessed by assigning a grade from 0 (worst) to 100 (best). In addition, the influence of preserving or not preserving the neurovascular bundles were investigated.

For comparison among the three different nervespared groups, one-way ANOVA and chi-square tests were used. Repeated measures ANOVA was performed for comparison of changes in the time-course UCLA-PCI among the different nerve-spared groups. Multiple regression analysis (stepwise method) was employed to investigate factors determining sexual function in the late postoperative phase. A significance level of less than 5\% was regarded as significant. Statistical analysis software (JMP version 7.0) was used. 


\section{RESULTS}

\section{Characteristics of patients by nerve preservation}

The characteristics of patients with and without nerve preservation are presented in Table 1. Significant differences were only observed in the preoperative PSA level and operation time. For the clinical stage which divided into those with localized (T1/T2) and non-localized (T3/T4) cases. Many patients in the non-spared group (T3-4: 11) had lesions that were not localized, while only one T3-4 case was present in the unilateral nerve-spared group, showing a significant difference $(\mathrm{p}<0.001)$. On the preoperative UCLAPCI survey there was no significant difference in any of the indices among the three different groups.
Changes in UCLA-PCI in groups with and without nerve preservation

Time-course changes in urinary function (A) and bother $(B)$ in groups with and without nerve preservation (Fig. 1): Regarding 'urinary function', there was no significant difference in the preoperative values. In the early postoperative phase, the value deteriorated in the bilateral nerve-spared group, but recovered to the preoperative level in the late postoperative phase. 'Urinary bother' slightly increased with changes in 'urinary function' in the early postoperative phase in all 3 groups, compared to that before surgery, but improved to a level higher than the preoperative value in the late postoperative phase. However, these timecourse changes or differences among the groups were not significant.

TABLE 1.

Characteristics of Patients by Group

\begin{tabular}{|c|c|c|c|c|c|}
\hline & & \multicolumn{3}{|c|}{$\begin{array}{l}\text { Preservation of nerves } \\
\text { Total }(n=105)\end{array}$} & \multirow{2}{*}{ Statistics } \\
\hline & & $\begin{array}{l}\text { none } \\
(\mathrm{n}=36)\end{array}$ & $\begin{array}{c}\text { unilateral } \\
(\mathrm{n}=24)\end{array}$ & $\begin{array}{c}\text { bilateral } \\
(\mathrm{n}=45)\end{array}$ & \\
\hline Age (year) & & 67.7 & 66.6 & 66.8 & n.s. \\
\hline Pre-operative PSA (ng/ml) & & 13.9 & 16.1 & 9.5 & $\mathrm{p}=0.003$ \\
\hline \multirow{2}{*}{$\begin{array}{l}\text { Clinical Stage } \\
\text { (before treatment of MAB) }\end{array}$} & $\mathrm{T} 1-2$ & 25 & 23 & 45 & \multirow{3}{*}{$\mathrm{p}<0.001$} \\
\hline & T3-4 & 11 & 1 & 0 & \\
\hline No. of MAB & & 13 & 3 & 3 & \\
\hline Operation Time (min) & & 165.9 & 166.2 & 150.5 & $\mathrm{p}=0.023$ \\
\hline Blood Loss (ml) & & 480.8 & 444.5 & 457.1 & n.s. \\
\hline \multirow{3}{*}{$\begin{array}{l}\text { Histological } \\
\text { Differentiation }\end{array}$} & well & 5 & 2 & 11 & \multirow{3}{*}{ n.s. } \\
\hline & moderately & 23 & 19 & 24 & \\
\hline & poorly & 1 & 1 & 5 & \\
\hline \multirow{3}{*}{$\begin{array}{l}\text { Pathological } \\
\text { Stage }\end{array}$} & T0-1 & 4 & 2 & 4 & \multirow{3}{*}{ n.s } \\
\hline & $\mathrm{T} 2$ & 27 & 17 & 30 & \\
\hline & $\mathrm{T} 3$ & 0 & 5 & 8 & \\
\hline \multirow{6}{*}{$\begin{array}{l}\text { UCLA-PCI scale } \\
\text { (pre-operative) }\end{array}$} & Urinary function & 93.9 & 89.7 & 86.8 & n.s. \\
\hline & Urinary bother & 84.7 & 90.6 & 87.8 & n.s. \\
\hline & Bowel function & 92.1 & 95.8 & 89.7 & n.s. \\
\hline & Bowel bother & 93.8 & 94.8 & 94.9 & n.s. \\
\hline & Sexual function & 24.9 & 38.9 & 34.0 & n.s. \\
\hline & Sexual bother & 77.1 & 79.2 & 73.3 & n.s. \\
\hline
\end{tabular}

Some data on histological differentiation and pathological stage were not available. n.s.: not significant 
(A)

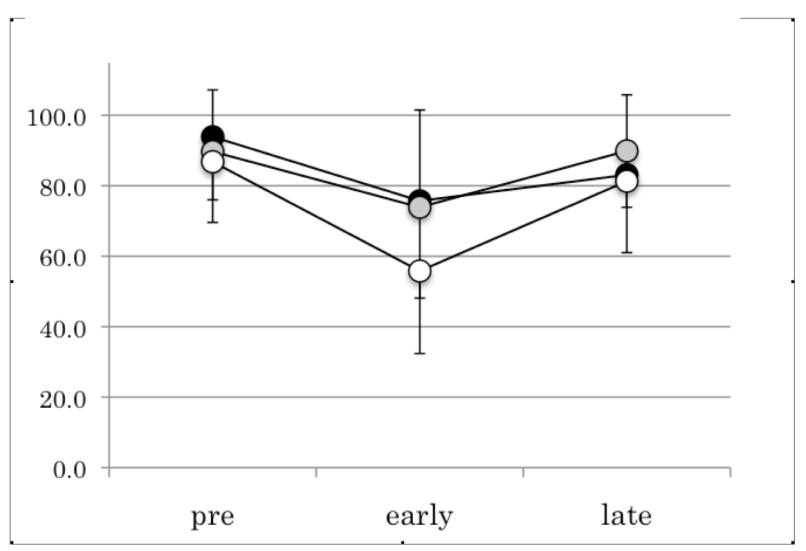

(B)

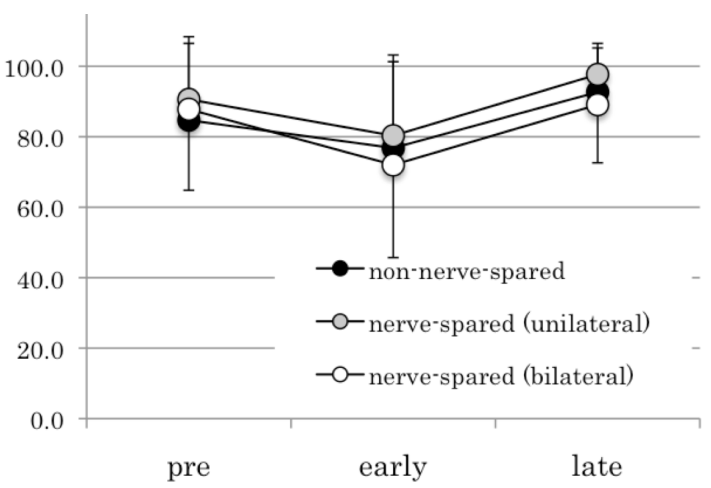

Fig. 1. Time-course changes in urinary function (A) and urinary bother (B) by nerve preservation. Urinary function and urinary bother were assessed by assigning a grade from 0 (worst) to 100 (best). Points of each symbol and vertical bar (one-side) indicate means and standard deviations, respectively.

Time-course changes in bowel function $(A)$ and bother $(B)$ in groups with and without nerve preservation (Fig. 2): Both 'bowel function' and 'bowel bother' slightly deteriorated in the early postoperative phase with or without nerve preservation, but recovered to the preoperative levels by the late postoperative phase in all three groups. There were no significant differences in the time-course changes between the groups.

Time-course changes in sexual function $(A)$ and bother $(B)$ in groups with and without nerve preservation (Fig. 3): Regarding 'sexual function', there were no preoperative differences among the three groups. In the early postoperative phase the mean scores dramatically decreased in all 3 groups, showing a marked impairment of the QOL. In the late postoperative phase, recovery was favorable in the bilateral nerve-spared group, but poor in the unilateral nervespared group as well as the non-nerve-spared group. The time-course changes in sexual function showed significant differences among the three groups ( $\mathrm{p}=0.0015$ on repeated-measures ANOVA). Regarding 'sexual bother', no aggravation was noted in the early postoperative phase in the non-nerve-spared group. In the nerve-spared group, despite an improved QOL score for 'sexual function', bother deteriorated in the late postoperative phase in the unilateral nervespared group, but the time-course change was not sig-
(A)

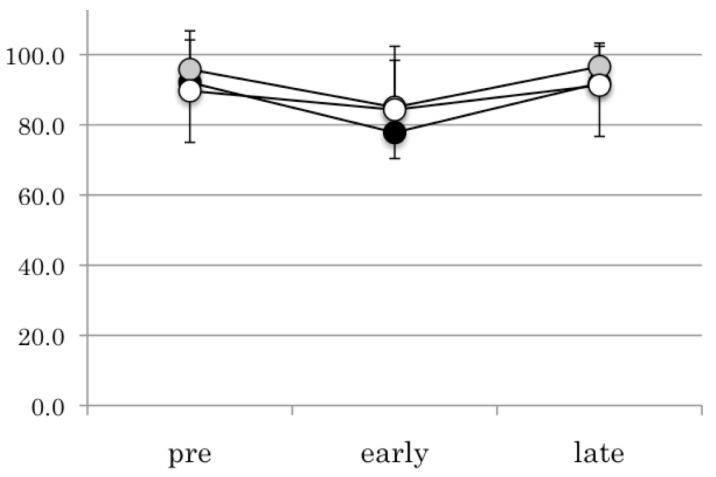

(B)

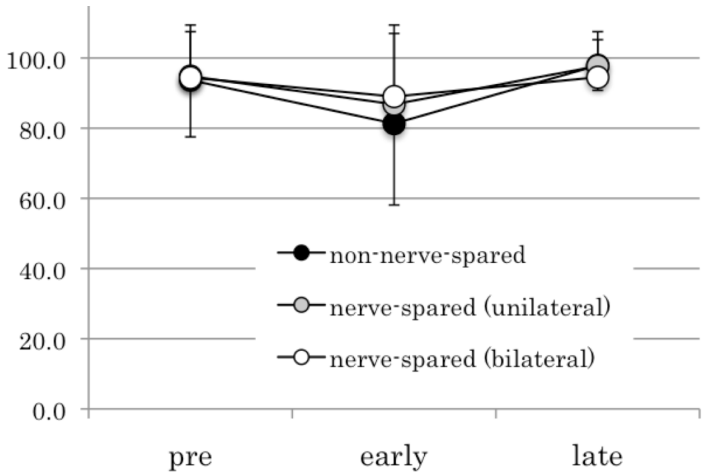

Fig. 2. Time-course changes in bowel function (A) and bowel bother (B) by nerve preservation. Bowel function and bowel bother were assessed by assigning a grade from 0 (worst) to 100 (best). Points of each symbol and vertical bar (one-side) indicate means and standard deviations, respectively. 
(A)

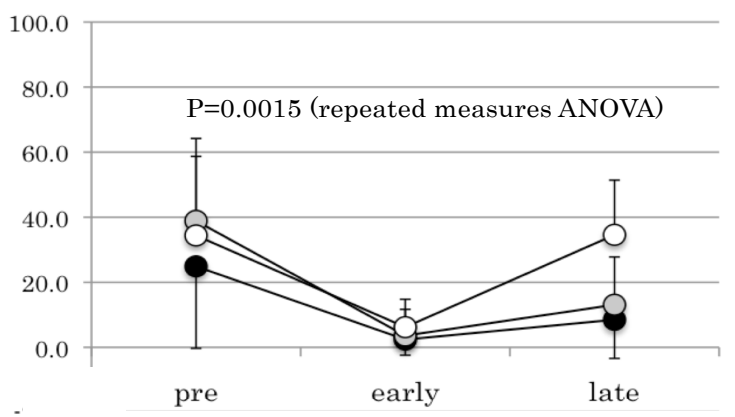

(B)

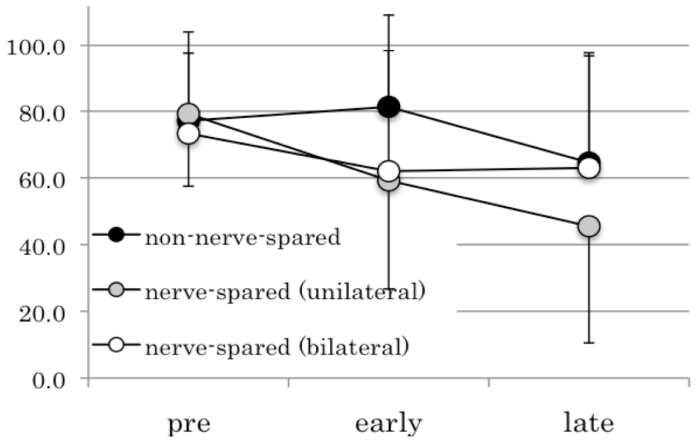

Fig. 3. Time-course changes in sexual function (A) and sexual bother (B) by nerve preservation. Sexual function and sexual bother were assessed by assigning a grade from 0 (worst) to 100 (best). Points of each symbol and vertical bar (one-side) indicate means and standard deviations, respectively.

nificant.

\section{Multiple regression analysis of sexual function}

Of the measured indices of the UCLA-PCI, a significant difference was noted in 'sexual function' in the late postoperative phase between the nerve-spared and non-spared groups. This finding was subjected to multiple regression analysis (stepwise method) regarding age, preoperative PSA level, operation time, blood loss, clinical stage (categorized into 2 stages), presence or absence of nerve preservation, and preoperative value of sexual function (on the UCLA-PCI scale) as explanatory variables. Nerve preservation and preoperative sexual function value (on the UCLAPCI scale) remained in the final model (Table 2). Only bilateral nerve preservation was significantly

TABLE 2.

Multiple Regression Analysis (UCLA-PCI Scale) of Late Postoperative Sexual Function

\begin{tabular}{cccc}
\hline & $\begin{array}{c}\text { Parameter } \\
\text { estimate }\end{array}$ & $\begin{array}{c}\text { Standard } \\
\text { error }\end{array}$ & $\begin{array}{c}\mathrm{p} \\
\text { value }\end{array}$ \\
\hline $\begin{array}{c}\text { Nerve-spared } \\
\text { [unilateral] } \\
\text { [bilateral] }\end{array}$ & -6.033 & 3.206 & 0.0653 \\
\hline $\begin{array}{c}\text { Pre-operative } \\
\text { sexual function }\end{array}$ & 0.124 & 0.0735 & 0.098 \\
\hline
\end{tabular}

Multiple regression analysis was performed by step-wise method (forward selection) for 7 variables (age, preoperative PSA level, operation time, blood loss in operation, clinical stage, nerve preservation and preoperative value of sexual function). associated with sexual function in the late postoperative phase $(\mathrm{p}<0.0001)$, whereas unilateral nerve preservation showed no association $(\mathrm{p}=0.0653)$.

\section{DISCUSSION}

Changes in the QOL following retropubic prostatectomy for localized prostate cancer were prospectively investigated using the UCLA-PCI. The UCLAPCI items 'urinary function', 'urinary bother', 'bowel function', and 'bowel bother', tended to deteriorate in the early postoperative phase (1-3 months after surgery), but recovered to the preoperative levels in the late postoperative phase (6-12 months after surgery), and there were no significant differences in the timecourse changes between the nerve-spared and nonspared groups. Regarding 'sexual function', the value on the QOL scale decreased in the early postoperative phase in both groups, but recovered in the late postoperative phase only in the nerve-spared group, showing a significant improvement of sexual function compared to that in the non-nerve-spared group. On multiple regression analysis to investigate factors associated with sexual function in the late postoperative phase, only the presence or absence of spared nerve showed a significant association.

Since Walsh et al. initially reported nerve-sparing radical prostatectomy [3], there have been many reports on sexual function following this surgery. Quinlan et al. surveyed patients with preoperative capability of sexual intercourse at least 18 months after surgery in a single facility, and found that $68 \%$ had sufficient potency for sexual intercourse [5]. In the study reported by Walsh et al., potency recovered in 
$73 \%$ of patients after 12 months and $86 \%$ after 18 months, showing favorable outcomes [6]. Catalona et al. reported that recovery of potency was favorable in young patients who underwent bilateral nerve-sparing surgery [7]. However, Litwin et al. reported that there was no difference in postoperative sexual function between patients with and without application of nerve-sparing [8]. Furthermore, Talcott et al. reported that recovery of postoperative potency in nerve-spared patients were less than previously reported [9]. With regard to the favorable outcomes achieved by Walsh et al., 1/3 of their patients were treated with Viagra (sildenafil), and it was pointed out that patient selection was involved in the outcome of recovered sexual function $[8,9]$. In a review of sexual function following radical prostatectomy reported by Dubbelman et al., the rate of patients with potency following bilateral nerve-sparing radical prostatectomy was within a range of $31-86 \%$, and the number of spared nerves, age, and preoperative sexual activity were the most important factors for the recovery of potency [10]. There is a marked variation in potency following nerve-sparing surgery among facilities, as shown above. Regarding the cause for this variation, Walsh et al. assumed that the variation was due to the weight of excised specimen, experience of the surgeon, and age and health condition of patients [6]. We analyzed factors associated with sexual function in the late postoperative phase by multiple regression analysis, and found no association with age, operation time, blood loss, preoperative PSA level, clinical stage (categorized into 2 stages), or preoperative value of sexual function on the UCLA-PCI scale. Only nerve sparing was significantly associated with sexual function. However, a marked difference due to the number of spared nerves (unilateral or bilateral) was noted, as reported by Dubbelman et al. Regarding this point, Noldus et al. [11] reported that recovery of sexual function was significantly more favorable in the bilateral nerve-spared than in the unilateral nerve-spared group. Rabbani et al. [12] also reported that the number of spared nerves was an influential factor for the postoperative recovery of sexual function.

Nerve preservation is an important factor in consideration of patients' QOL following radical prostatectomy, and this study clarified that bilateral nerve sparing is desirable. Nerve-sparing is an important independent factor for the recovery of sexual function following radical prostatectomy, separate from the other factors, such as age and preoperative sexual function, supporting the recommendation of nerve-sparing surgery, but it may be wise to use electric stimulation to accurately judge the feasibility of nerve-sparing. The results of this study may be useful in explaining surgical procedures and the effects of radical prostatectomy to patients.

We used the UCLA-PCI for the QOL questionnaire. The UCLA-PCI is a disease-targeted QOL questionnaire, covering 'urinary function', 'urinary bother', 'bowel function', 'bowel bother', 'sexual function', and 'sexual bother' as typical complications following radical prostatectomy. It is simple, consisting of only 20 items, and requires little time or effort on the part of patients, so it is less stressful than other indices [4]. However, QOL factors other than sexual function may also determine the recovery of potency, as Litwin et al. reported that sexual function was impaired by the presence of urinary incontinence in $20 \%$ of patients who underwent surgery [8]. Moreover, inconvenience in daily life, not only the age of the patient but also the age and sexual activity of his partner, and patient's mental stress may affect sexuality and potency. Thus, for the assessment of postoperative sexual function, the QOL should be comprehensively evaluated in various aspects by concomitantly employing FACT-P, EORTC, and SF-36 as general health-related QOL questionnaires.

Distress tended to increase in the late postoperative phase in the unilateral nerve-spared group. The patients may have been anxious about when their function would recover to the preoperative level, or may have had unrealistic expectation for the spared nerve due to inadequate explanation before surgery of the likelihood of recovery of sexual function by unilateral nerve-sparing. This was a matter of concern regarding the suitability of radical prostatectomy for localized prostate cancer, reconfirming that changes in the QOL after surgery should be considered in the evaluation of the prognosis, and detailed information concerning sexual function, including possible changes in the QOL after surgery should be provided to patients before surgery.

On the UCLA-PCI scale, the value of sexual function was 32.2 before surgery, and those in the early and late postoperative phases were 4.0 and 19.7, respectively. These were markedly lower than those in patients following open radical prostatectomy reported by Ball et al. [13], where sexual function on the UCLA-PCI was 59 before surgery, and 19, 24, and 33 at 1,3, and 6 months after surgery. These differences may reflect the fact that reduced sexual function and activity is not regarded as so important in the daily life of the elderly in Japan as it seems to be in Western countries. It is also implied that differences 
in ways of thinking or habits concerning sex between Europeans/Americans and Japanese may be involved. Since there have been no reports concerning Japanese sexual culture, further assessment of the QOL with regard to 'sex' in Japanese may be necessary.

\section{REFERENCES}

1. Hamada Y, Kitani K, Kawano T, Otsuka Y, Sasho K et al. Assessment of quality of life in men treated for localized prostate cancer: before and after radical prostatectomy. Nishinihon J Urol 2004; 66:241-248.

2. Noguchi M, Shimada A, Yahara J, Suekane S, and Noda SA. Early catheter removal 3 days after radical retropubic prostatectomy. Int J Urol 2004; 11:983-988.

3. Walsh PC, and Donker PJ. Impotence following radical prostatectomy: insight into etiology and prevention. J Urol 1982; 128:492-497.

4. Suzukamo Y, Kakei Y, Kamamoto T, Arai Y, Ogawa O et al. Translation and adaptation of the UCLA Prostate Cancer Index for use in Japan. Jpn J Urol 2002; 93:659-668. (in Japanese)

5. Quinlan DM, Epstein JI, Carter BS, and Walsh PC. Sexual function following radical prostatectomy: Influence of preservation of neurovascular bundles. J Urol 1991; 145:998-1002.

6. Walsh PC, Marschke P, Ricker D, and Burnett AL. Patientreported urinary continence and sexual function after ana- tomic radical prostatectomy. Urology 2000; 55:58-61.

7. Catalona WJ, Carvalhal GF, Mager DE, and Smith DS. Potency, continence and complication rates in 1,870 consecutive radical retropubic prostatectomies. J Urol 1999; 162:433-438.

8. Litwin MS, Hays RD, Fink A, Ganz PA, Leake B et al. Quality-of-life outcomes in men treated for localized prostate cancer. JAMA 1995; 273:129-135.

9. Talcott JA, Rieker P, Propert KJ, Clark JA, Wishnow KI et al. Patient-reported impotence and incontinence after nerve-sparing radical prostatectomy. J Natl Cancer Inst 1997; 89:1117-1123.

10. Dubbelman YD, Dohle GR, and Schroder FH. Sexual function before and after radical retropubic prostatectomy: A systematic review of prognostic indicators for a successful outcome. Eur Urol 2006; 50:711-720.

11. Noldus J, Michl U, Graefen M, Haese A, Hammerer P et al. Patient-reported sexual function after nerve-sparing radical retropubic prostatectomy. Eur Urol 2002; 42:118-124.

12. Rabbani F, Stapleton AM, Kattan MW, Wheeler TM, and Scardino PT. Factors predicting recovery of erections after radical prostatectomy. J Urol 2000; 164:1929-1934.

13. Ball AJ, Gambill B, Fabrizio MD, Davis JW, Given RW et al. Prospective longitudinal comparative study of early health-related quality-of-life outcomes in patients undergoing surgical treatment for localized prostate cancer : A short-term evaluation of five approaches from a single institution. J Endourol 2006; 20:723-731. 